\title{
Room Temperature-Operating Spin-Valve Transistors Formed by Vacuum Bonding
}

\author{
D. J. Monsma,* R. Vlutters, J. C. Lodder
}

\begin{abstract}
Functional integration between semiconductors and ferromagnets was demonstrated with the spin-valve transistor. A ferromagnetic multilayer was sandwiched between two device-quality silicon substrates by means of vacuum bonding. The emitter Schottky barrier injected hot electrons into the spin-valve base. The collector Schottky barrier accepts only ballistic electrons, which makes the collector current very sensitive to magnetic fields. Room temperature operation was accomplished by preparing Si-Pt-Co-Cu-Co-Si devices. The vacuum bonding technique allows the realization of many ideas for vertical transport devices and forms a permanent link that is useful in demanding adhesion applications.
\end{abstract}

Ten years after its discovery (1), giant magnetoresistance (GMR) or, designated more appropriately, the spin-valve effect, has already shown its strength in applications such as read heads and magnetic random access memories (MRAMs). Driven by such lowfield applications, a search for higher sensitivities is continuing. In the spin-valve effect, majority carrier electrons with long mean free paths can travel with low resistance through a multilayer when a magnetic field aligns magnetizations of adjacent magnetic layers. In ordinary four-point resistance measurements with the current in plane (CIP), channeling, shunting, and diffusive surface scattering diminish and complicate the effect. Experiments with currents perpendicular to the planes (CPP) are very useful for fundamental studies of the electron transport process (2), yet application of the larger effect to sensors is cumbersome because of the very low resistances involved. We introduced the solidstate spin-valve transistor (SVT) structure as a spectroscopic tool to investigate transport properties of the CPP spin-valve effect and found a large, perpendicular, hot-electron spin-valve effect (3). In addition, we proposed to employ nonmagnetic and ferromagnetic tunnel barriers, of which experimental results were reported later (4). Such experiments had to be conducted at $77 \mathrm{~K}$ to decrease the collector leakage current with respect to the hot-electron current. Room-temperature (RT) operation requires a large emitter current density, reduction of the collector barrier area, increase of the collector barrier height, and enhancement of the base transport factor. In order to obtain sufficiently large

MESA Research Institute, University of Twente, 7500AE Enschede, Netherlands.

*To whom correspondence should be addressed. Present address: IBM Almaden Research, 650 Harry Road, San Jose, CA 95120, USA. injection current densities, a device-quality semiconductor layer would need to be grown on top of the metallic base. In practice, such layers grow poorly, so we instead turned to bonding the base between two crystalline substrates in air. This provided spontaneous adhesion based on van der Waals forces and hydrogen bonds between adsorbed water molecules. This bond was not strong enough, however, to allow lithographic processing. Our development of vacuum metal bonding during sputtering provided a superior bond and hot-electron transport in a nonmagnetic $\mathrm{Si}-\mathrm{Au}-\mathrm{Ge}$ metal base transistor (5). We have used this technique to prepare SVTs operating at RT. Because the RT operation now also permits important magnetic-field sensor applications, we compared the signal-to-noise ratio (SNR) of this device to a state-of-the-art CIP spin-valve sensor.

The SVT (Fig. 1A) was formed as follows. A Co-Cu-Co-Pt multilayer was sandwiched between two Si (100) substrates; the emitter was negatively biased (forward) with a dc current source, and the collector substrate was in reverse (positive bias). A schematic energy band diagram is shown in Fig. 1B. The emitter bias accelerates the electrons over the emitter metal-semiconductor (Schottky) barrier, after which they constitute the perpendicular hot electrons in the base. The number of electrons crossing the base is spin-dependent and is described with a modified CPP-GMR two-channel model (3), resulting in the transmission equation $I_{\mathrm{P}(\mathrm{AP})}=$ $I_{\mathrm{E}} \cdot T_{\mathrm{SI}} \cdot 1 / 2 T_{\mathrm{P}(\mathrm{AP})}+I_{\mathrm{L}}$, where $I_{\mathrm{P}(\mathrm{AP})}$ is the collector current for parallel or antiparallel magnetizations, $I_{\mathrm{E}}$ is the emitter current, $T_{\mathrm{SI}}$ $=\alpha_{\mathrm{e}} \alpha_{\mathrm{c}} \alpha_{\mathrm{qm}} e^{\frac{-W_{\mathrm{nm}}}{\lambda_{\mathrm{mm}}}}$ is the spin-independent ballistic transmission probability (BTP) factor, and $I_{\mathrm{L}}$ is the leakage current. Here $\alpha_{\mathrm{e}} \alpha_{\mathrm{c}} \alpha_{\mathrm{qm}}$ $\approx 0.1$ represents BTP factors reduced by emitter and collector optical phonon scatter- ing and quantum mechanical reflections at the collector barrier and $e^{\frac{-W_{\mathrm{mm}}}{\lambda_{\mathrm{m}}}}$ represents the BTP through the nonmagnetic layers in the base ( $\mathrm{Pt}$ and $\mathrm{Cu}$ ). $T_{\mathrm{P}(\mathrm{AP})}$ is the sum of spin-up and spin-down BTPs for parallel or antiparallel magnetizations and may be written in a simplified form for the $\mathrm{Co} / \mathrm{Cu} / \mathrm{Co}$ sandwich as $T_{-W_{\mathrm{P}}}=e^{\frac{-W_{\mathrm{C}_{0}} \uparrow}{\lambda_{\mathrm{C}}}}+e^{\frac{-W_{\mathrm{C} 0}}{\lambda_{\mathrm{CO} \downarrow}}}$ and $T_{\mathrm{AP}}=2 e^{\frac{-W_{\mathrm{C}}}{2 \lambda_{\mathrm{C} 0}}}$. $e_{2 \lambda_{\mathrm{Co} \downarrow} \downarrow}$, where $\lambda_{\mathrm{Co} \uparrow(\downarrow)}$ is the spin-up or spindown mean free path in the Co, and $W_{\mathrm{Co}}$ is the total Co thickness. Because some electrons in the base have sufficient thermal energy to surmount the reverse-biased collector barrier, they form a parasitic $I_{\mathrm{L}}=110 \mathrm{~A}$. $T^{2} e^{\frac{-q \phi_{c}}{k T}}$ (6). Here $A$ is the collector area in square centimeters and $\phi_{\mathrm{c}}$ is the collector barrier height. The exponential dependence of the collector current $I_{\mathrm{C}}$ on the mean free paths in the base is a result of the small acceptance angle of the collector barrier, as described for nonmagnetic metal base transistors (6) and ballistic electron emission microscopy (7) with a gold base: A single scattering event deflects the electron out of the acceptance angle of the collector. This effect causes the relative change in $I_{\mathrm{C}},\left(I_{\mathrm{P}}-I_{\mathrm{AP}}\right) /$ $I_{\mathrm{AP}}$, to increase strongly with the thickness of the magnetic layer as well as with $\lambda_{\uparrow} / \lambda_{\downarrow}$, which is the mean free path asymmetry of majority and minority electrons. The energy of the electrons in the multilayer can be varied by altering the Schottky barrier height (or a tunnel injector bias).

Prime quality $n$-type $\operatorname{Si}(100)$ wafers were used to prepare the SVTs. After cleaning, the two substrates (about $1 \mathrm{~cm}$ by $2 \mathrm{~cm}$ in size) were attached to the rotatable arms of a bond tool with clips. The $\mathrm{Co}-\mathrm{Cu}$ sandwich was sputtered on one substrate (background pressure $\sim 10^{-8}$ mbar). During subsequent sputtering of Pt on both substrates, the substrates
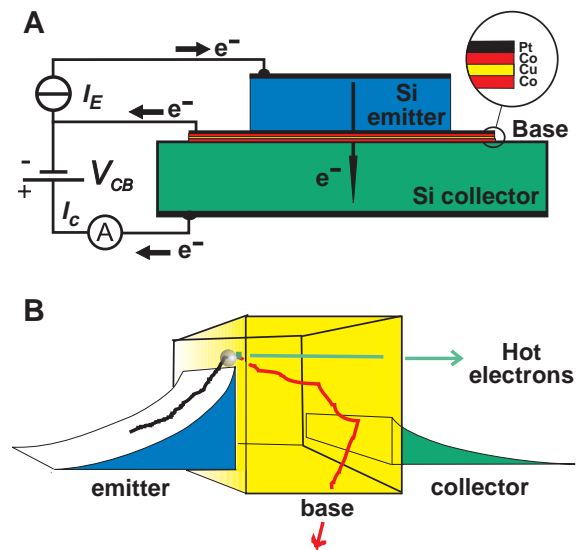

Fig. 1. (A) Schematic cross section and (B) schematic energy band diagram of the spinvalve transistor under forward-bias emitter Schottky barrier and the reverse-biased collector Schottky barrier as well as ballistic and scattered electron transport. 


\section{REPORTS}

Fig. 2. (A) Cross-sectional TEM image of Si-Co-Cu-Co-Pt-Si SVTs demonstrating the spontaneous formation of a continuous Pt film during room-temperature vacuum bonding and (B) scanning electron micrograph after lithographic processing, displaying different device areas.
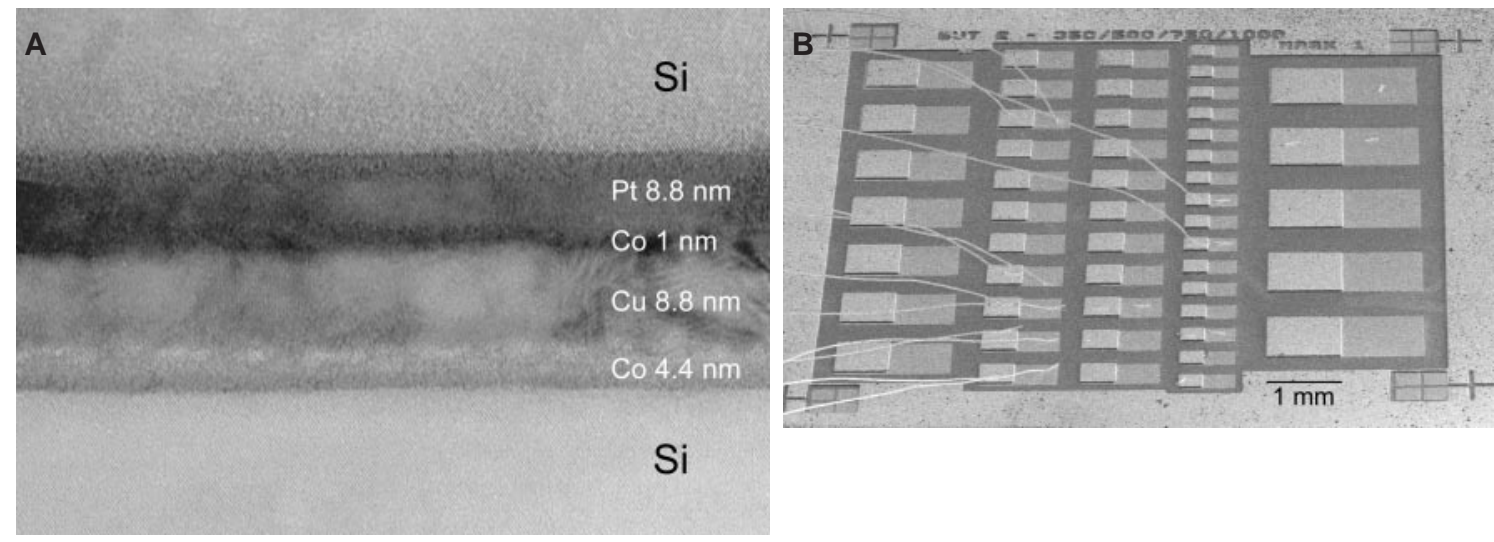

were rotated toward each other, and the two freshly sputtered Pt surfaces came into contact. Bonding reduces their surface free energies, but stress created by roughness compensation during matching of the surfaces counteracts this drive. For microroughnesses smaller than about $2 \mathrm{~nm}$, complete bonding proceeded spontaneously, that is, without force at RT. Additional energy may be extracted from the sputter energy: The adatoms typically arrive with an excess energy of about 1 to $20 \mathrm{eV}(8)$. The resulting bonding strength was greater than the fracture strength of the silicon. The yield of the bonding procedure is surprisingly good $(100 \%$ under standard conditions). High-resolution transmission electron microscopy (TEM) images

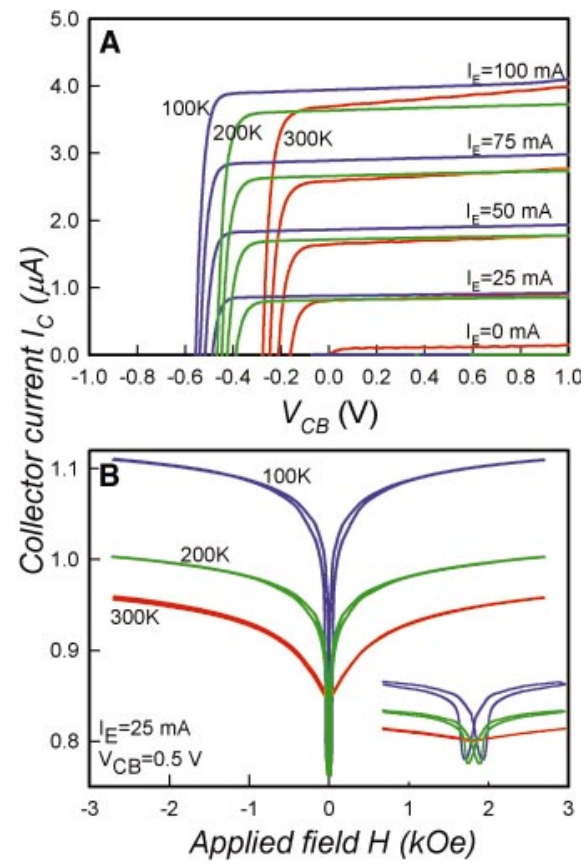

Fig. 3. (A) Common base characteristics of the Co-Cu-Co-Pt SVT with $V_{\mathrm{CB}}$ and $I_{\mathrm{E}}$ as indicated in Fig. 1A. (B) Collector current versus applied magnetic field for $T=100,200$, and $300 \mathrm{~K}$. The relative change in collector current is 42,32 , and $15 \%$, respectively. The inset accentuates small field behavior; the field range is -300 to 300 Oe. of these vacuum-bonded metals show either grain boundary-like interfaces $(\mathrm{Pt})$ or complete absence of an interface (Ti) because of recrystallization induced by interface energy minimization (9). A TEM image of the Co$\mathrm{Cu}-\mathrm{Co}-\mathrm{Pt} \mathrm{SVT}$ is shown in Fig. 2A. For RT operation of the SVT, the leakage current was decreased by replacing the $\mathrm{Cu}$ collector barrier by $\mathrm{Co}$ and by making the collector area smaller. Moreover, a Co-Cu-Co sandwich replaced the $(\mathrm{Co}-\mathrm{Cu})_{4}$ multilayer base to increase the base transmission. Vacuum-bonded Si-Co-Cu-Co-Pt-Si SVTs with different device areas obtained after photolithography and etching are shown in Fig. 2B. Platinum was used to induce a barrier height difference between emitter and collector in order to minimize quantum mechanical reflections.

The CIP-magnetoresistance (MR) of the $\mathrm{Co}(4.4 \mathrm{~nm})-\mathrm{Cu}(8.8 \mathrm{~nm})-\mathrm{Co}(1 \mathrm{~nm})-\mathrm{Pt}(8.8$ $\mathrm{nm}$ ) sandwich in the SVT showed $0.1 \% \mathrm{MR}$ at $300 \mathrm{~K}$ and $0.3 \%$ at $77 \mathrm{~K}$. The CIP-MR was small because of shunting and channeling in the relatively thick $\mathrm{Cu}$ layer, diffusive surface scattering, and incomplete antiferromagnetic

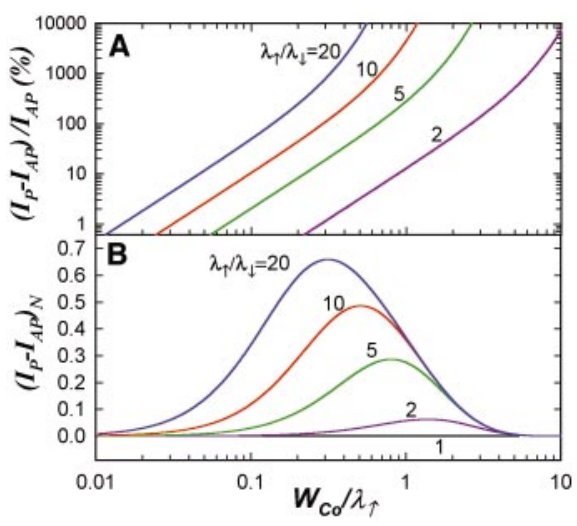

Fig. 4. (A) The relative collector current change $\left(I_{\mathrm{P}}-I_{\mathrm{AP}}\right) / I_{\mathrm{AP}}$ and (B) the (normalized) absolute change in collector current $\left(I_{\mathrm{P}}\right.$ $\left.I_{\mathrm{AP}}\right)_{\mathrm{N}}$ plotted versus the base transport factor $W_{\mathrm{Co}} / \lambda_{\uparrow}$ for different $\lambda_{\uparrow} / \lambda_{\downarrow}$ values. Here $W_{C o}$ is the total Co thickness and $\lambda_{\uparrow, \downarrow}$ is the spin-dependent mean free path. For small $W_{\mathrm{Co}_{0}} /$ $\lambda_{\uparrow}$ and large $\lambda_{\uparrow} / \lambda_{\downarrow},\left(I_{\mathrm{P}}-I_{\mathrm{AP}}\right)_{\mathrm{N}} \rightarrow 1$ and $I_{\mathrm{P}}-I_{\mathrm{AP}}$ approaches $1 / 2 I_{\mathrm{E}} T_{\mathrm{SI}}$.
(AF) alignment caused by poor growth (silicide formation) on oxide-free silicon (Fig. 2A). Measurements of the emitter and collector Schottky barriers of the SVT showed barrier heights of 0.8 and $0.7 \mathrm{eV}$ and ideality factors of 1.14 and 1.07 at $300 \mathrm{~K}$, respectively. The common base curves corresponding with the electrical setup of Fig. 1A are shown in Fig. 3A, measured at saturation field (1 T). At $300 \mathrm{~K}$, the curve with $I_{\mathrm{E}}=0$ shows the characteristic of the collector barrier and its reverse characteristics for $V_{\mathrm{CB}}>0 \mathrm{~V}$. The collector leakage current $I_{\mathrm{L}}$ is about $0.1 \mu \mathrm{A}$, which is saturating as expected and is much smaller than the hot-electron currents, even at $300 \mathrm{~K} . I_{\mathrm{C}}$ follows the injected emitter current linearly, as expected from the transport equations. On cooling down, $I_{\mathrm{C}}$ first decreases because of the rapid decrease of $I_{\mathrm{L}}$. Then, after $I_{\mathrm{L}}$ is reduced to negligible values, $I_{\mathrm{C}}$ rises again because of the increased mean free paths in the base and semiconductors. As a result of the perpendicular trajectory, the exponential mean free path dependence, and possibly also the electron energy, the variation in $I_{\mathrm{C}}$ is large (Fig. 3B). In this graph, $I_{\mathrm{C}}$ is plotted versus applied magnetic field for an injection current of $25 \mathrm{~mA}$ and reverse bias of $0.5 \mathrm{~V}$. At zero field, $I_{\mathrm{C}}$ is small because antiparallel magnetizations block both spinup and spin-down current. At large fields, the magnetizations of the two Co layers align and result in an enlarged (majority spin) current. The effect is $15 \%$, which is a factor of 150 greater than for the CIP case. Nevertheless, taking $T_{\mathrm{SI}}=0.01$ (0.01 is a typical transmission factor in a metal base transistor with a 9-nm Pt film), $W / \lambda_{\uparrow}$ is about 5.5. The ratio $\lambda_{\uparrow} / \lambda_{\downarrow}$ is smaller than 2 (Fig. 4A), which we attribute to incomplete antiparallel ordering at zero field. Lowering the temperature increases the AF alignment, which is evidenced by the decreased $I_{\mathrm{c}}\left(H_{\mathrm{c}}\right)$ at 200 and $100 \mathrm{~K}$, whereas the current is greater at saturation. The $\left(I_{\mathrm{P}}-I_{\mathrm{AP}}\right) / I_{\mathrm{AP}}$ results are not affected by applied emitter current, because the barrier height and electron energy of a Schottky barrier are not affected by applied bias.

By using proper spin-valve sandwiches in 


\title{
R E P O R T S
}

the base, the relative change in collector current $\left(I_{\mathrm{P}}-I_{\mathrm{AP}}\right) / I_{\mathrm{AP}}$ can be greater than $1000 \%$, depending on the scattering asymmetry $\lambda_{\uparrow} / \lambda_{\downarrow}$ and $W_{\mathrm{Co}} \lambda_{\uparrow}$ (Fig. 4A). In general, such large changes may not be necessary. The noise in the SVT is caused mainly by three sources: (i) (particle-related) shot noise from the forwardbiased emitter barrier; (ii) ordinary thermal, or Johnson, noise in the base resistance; and (iii) shot noise in the collector barrier. These fluctuations produce white noise at the terminals of the SVT. Because the thermal noise of electrons at $E_{\mathrm{F}}$ does not affect hot-electron transport, and the base thermal noise voltage does not influence the hot-electron $I_{\mathrm{C}}$ (the emitter is driven by a current source), the thermal noise is not found in $I_{\mathrm{C}}$. Hence, the collector noise current $i_{\mathrm{c}}$ is pure shot noise $i_{\mathrm{c}}=\left(2 q I_{\mathrm{c}} d f\right)^{1 / 2}$ as in ordinary (Schottky) diodes (10). For this reason, the SNR increases with $I_{\mathrm{C}}$ and the absolute change in collector current $I_{\mathrm{P}}-I_{\mathrm{AP}}$ is a more useful parameter for sensor applications than the relative change $\left(I_{\mathrm{P}}-I_{\mathrm{AP}}\right) / I_{\mathrm{AP}}$. Because the output current decreases with $W_{\mathrm{Co}} \lambda_{\uparrow}$ but the relative change increases with $W_{\mathrm{Co}} / \lambda_{\uparrow}$ and $\lambda_{\uparrow} / \lambda_{\downarrow}$, an optimum for the SNR is found by plotting $I_{\mathrm{P}}-$ $I_{\mathrm{AP}}$ versus $W_{\mathrm{Co}} \lambda_{\uparrow}$ (Fig. 4B). Here,

$$
\left(I_{\mathrm{P}}-I_{\mathrm{AP}}\right)_{\mathrm{N}}=\frac{\mathrm{I}_{\mathrm{P}}-I_{\mathrm{AP}}}{1 / 2 I_{\mathrm{E}} T_{\mathrm{SI}}}=T_{\mathrm{P}}-T_{\mathrm{AP}}
$$

is the normalized collector current difference between parallel and antiparallel magnetizations resulting from the two-channel model. We compare the SVT with a GMR film-measured CIP on an SNR basis by assuming a device area $1 \mu \mathrm{m}$ by $1 \mu \mathrm{m}$, a bandwidth $d f$ of $100 \mathrm{MHz}$, $\lambda_{\uparrow} / \lambda_{\downarrow}=10, W_{\mathrm{Co}} / \lambda_{\uparrow}=1 \Rightarrow\left(I_{\mathrm{P}}-\mathrm{I}_{\mathrm{AP}}\right)_{\mathrm{N}} \approx$ $0.4, I_{\mathrm{E}}=1 \mathrm{~mA}$, and $T_{\mathrm{SI}}=0.05$ (loss is lower when a thinner Pt film is used, for example). For the SVT, the shot noise results in an SNR = $20 \log \left(I_{\mathrm{c}} / i_{\mathrm{c}}\right)=58 \mathrm{~dB}$. Addition of a typical low-noise broadband (100 MHz) current (transimpedance) amplifier to this signal adds 1 $\mathrm{pA} / \mathrm{Hz}^{1 / 2}$ noise, resulting in a noise level of $i_{\text {tot }}$ $=\left(i_{\mathrm{c}}{ }^{2}+i_{\mathrm{a}}{ }^{2}\right)^{1 / 2}$ or $\mathrm{SNR}=57 \mathrm{~dB}$. Clearly, a large collector current is desired, which can be obtained by optimizing $I_{\mathrm{P}}-I_{\mathrm{AP}}$ through $W_{\mathrm{Co}} / \lambda_{\uparrow}$ (type of metal and number of interfaces) and optimizing $\lambda_{\uparrow} / \lambda_{\downarrow}$ (spin-valve quality) and $T_{\mathrm{SI}}$ (Schottky barrier quality and nonmagnetic layer thickness). In epitaxial structures, spin-dependent resonance effects in the base might further enhance $I_{\mathrm{P}}-I_{\mathrm{AP}}$. In cases where the input noise current of the amplifier limits the SNR of the SVT or when local amplification is desired, a major enhancement may be achieved by avalanche multiplication of the collector current. Application of a larger $V_{\mathrm{CB}}$ bias generates electron-hole pairs in the collector depletion layer, and multiplication of $I_{\mathrm{C}}$ may be as large as 100 . The noise current of the SVT is also multiplied with this factor, therefore its SNR stays the same, but because the signal is much greater, the SNR of the system will be enhanced. A comparable 20-ohm GMR film with 1-mV ef- fective output voltage (11) has a Johnson noise of $u_{\mathrm{n}}=(4 k T R d f)^{1 / 2} \approx 6 \mu \mathrm{V}$ and an SNR of 45 dB. A typical hard disk amplifier adds 0.55 $\mathrm{nV} / \mathrm{Hz}^{1 / 2}$ or $5.5 \mu \mathrm{V}$ of noise, resulting in $42 \mathrm{~dB}$ overall. In spite of the larger SNR of the SVT, the power dissipated in the SVT structure is greater $(\approx 1 \mathrm{~mW})$. The maximum current through the GMR film is determined by electromigration, whereas the maximum current in the SVT is determined by heating. Analysis of this factor for specific sensor designs is required for a realistic comparison. Its intrinsic diode characteristics make selection transistors per storage cell redundant and make the SVT attractive for MRAM development.

References and Notes

1. M. N. Baibich et al., Phys. Rev. Lett. 61, 2472 (1988)

2. For a review, see M. A. M. Gijs and G. E. W. Bauer, Adv. Phys. 46, 285 (1997).
3. D. J. Monsma, J. C. Lodder, Th. J. A. Popma, B. Dieny, Phys. Rev. Lett. 74, 5260, (1995).

4. K. Mizushima, T. Kinno, T. Yamauchi, K. Tanak, IEEE Trans. Magn. 33, 3500 (1997).

5. D. J. Monsma et al., ibid., p. 3495.

6. S. M. Sze, Physics of Semiconductor Devices (WileyInterscience, New York, 1969), chap. 11; C. R. Crowell and S. M. Sze, in Physics of Thin Films, G. Haas and R. E. Thun, Eds. (Academic Press, New York, 1967), vol. 4

7. L. D. Bell and W. J. Kaiser, Phys. Rev. Lett. 61, 2368 (1988).

8. J. C. S. Kools, IEEE Trans. Magn. 32, 3165 (1996)

9. T. Shimatsu, R. Mollema, D. J. Monsma, E. G. Keim, J. C. Lodder, J. Vac. Sci. Tech. A, in press.

10. A. M. Cowley and R. A. Zettler, IEEE Trans. Electr. Dev. 15, 761 (1968)

11. J. A. Brug et al., J. App. Phys. 79, 4491 (1996).

12. Supported by the European Community [grants Brite Euram BRE2-0546 and MEL-ARI ESPRIT 23.307 (SPIDER)]. The authors gratefully acknowledge B. Dieny, A. Vedyaev, H. Wallinga, F. N. Hooge, and E. G. Keim.

30 March 1998; accepted 2 June 1998

\section{Boundary Formation in Drosophila Wing: Notch Activity Attenuated by the POU Protein Nubbin}

\author{
Carl J. Neumann* and Stephen M. Cohen $\dagger$
}

\begin{abstract}
Cell interactions mediated by Notch-family receptors have been implicated in the specification of tissue boundaries in vertebrate and insect development. Although Notch ligands are often widely expressed, tightly localized activation of Notch is critical for the formation of sharp boundaries. Evidence is presented here that the POU domain protein Nubbin contributes to the formation of a sharp dorsoventral boundary in the Drosophila wing. Nubbin represses Notchdependent target genes and sets a threshold for Notch activity that defines the spatial domain of boundary-specific gene expression.
\end{abstract}

Spatially localized activation of Notch is required for specification of the dorsoventral (DV) boundary of the Drosophila wing (15 ). Notch signaling has also been implicated in establishing tissue boundaries in somite formation, in neurogenesis, and at the DV boundary of the vertebrate limb $(6-8)$. The tight localization of Notch activity in these systems contrasts with the broad distribution of Notch ligands. The problem of spatially limiting Notch activation is partially solved through activity of fringe genes (9), which modulate the sensitivity of Notch for its ligands and contribute to spatially limiting Notch activity $(8,10)$. Certain features of the abnormal wings in flies mutant for the nubbin gene suggested a possible role for Nubbin

European Molecular Biology Laboratory, Meyerhofstrasse 1, 69117 Heidelberg, Germany.

*Present address: MPI for Developmental Biology, D-72076 Tubingen, Germany.

†To whom correspondence should be addressed. Email: scohen@embl-heidelberg.de protein in spatially limiting Notch activity at the DV boundary of the wing $(11,12)$. The nubbin gene encodes a POU domain protein that is expressed in the developing wing primordium (11) (Fig. 1A).

The row of sensory bristles that makes up the wing margin is disorganized in nubbin mutant wings (11), suggesting a defect in Wingless or Notch activity. In preparations where the wing margin is viewed edge on, this disorganization reflects a broadening of the region where bristles form (Fig. 1, B and C). Margin bristles are normally specified in cells very close to the DV boundary, reflecting a requirement for high levels of Wingless signaling activity (13). The broadening of the margin suggests that Wingless might be ectopically expressed in nubbin mutant wing discs. Wingless is normally expressed in a stripe of two to three cells straddling the DV boundary (Fig. 1D). In nubbin mutant discs this stripe is widened considerably (Fig. 1E). Expression of the Notch targets vestigial and cut is similarly expanded at the DV boundary 